\title{
MEAN-FIELD OPTIMAL CONTROL PROBLEM OF SDDES DRIVEN BY FRACTIONAL BROWNIAN MOTION
}

\author{
BY \\ SOUKAinA DOUISS I* (MARrakech), NACIRA AGRA M** (OSLO), AND \\ ASTRID HIL BERT (VÄXJö)
}

\begin{abstract}
We consider a mean-field optimal control problem for stochastic differential equations with delay driven by fractional Brownian motion with Hurst parameter greater than $1 / 2$. Stochastic optimal control problems driven by fractional Brownian motion cannot be studied using classical methods, because the fractional Brownian motion is neither a Markov process nor a semi-martingale. However, using the fractional white noise calculus combined with some special tools related to differentiation for functions of measures, we establish necessary and sufficient stochastic maximum principles. To illustrate our study, we consider two applications: we solve a problem of optimal consumption from a cash flow with delay and a linear-quadratic (LQ) problem with delay.
\end{abstract}

2020 Mathematics Subject Classification: Primary 60G22, 60H07, 60H40, 93E20; Secondary 91G80.

Key words and phrases: mean-field, stochastic delayed differential equations, fractional Brownian motion, stochastic maximum principles.

\section{INTRODUCTION}

The interest in stochastic delayed differential equations is constantly increasing. They are frequently used to model the evolution of systems with past-dependence nature. Such systems usually appear in biology, engineering and mathematical finance. There is a rich literature on stochastic optimal problems with delay. A lot of authors studied both the case where the stochastic systems are driven by a classical Brownian motion as well as where there are jumps: see, e.g., [9], [18], [17], [10].

${ }^{*}$ Research supported by the Erasmus + International Credit mobility between Linnaeus University and Cadi Ayyad University for the academic year 2016-2017.

** Research supported by the Norwegian Research Council, within the research project Challenges in Stochastic Control, Information and Applications (STOCONINF), project number 250768/F20. 
Stochastic control problems driven by fractional Brownian motion ( $\mathrm{fBm}$ ) were also studied by many authors: see, e.g., [2], [4], [14], [19]. However, compared with the papers on stochastic control problems driven by the classical Brownian motion, little has been done because classical methods to solve control problems cannot be used directly, since the fractional Brownian motion is not a semi-martingale or a Markov process. Mean-field problems have also attracted wide attention recently, due to their applications in physics, economics, finance and stochastic games. Mean-field games were first studied by Lasry and Lions [15]. Buckdahn, Li and Peng [5] studied special mean-field games and introduced the so-called mean-field backward stochastic differential equations. Later, Carmona and Delarue [8] studied a class of mean-field forward-backward stochastic differential equations and gave many applications.

In this paper, all the previous fields are combined to study the optimal control problem of mean-field delayed stochastic differential equations driven by fractional Brownian motion. The dynamic of the controlled state process depends on the state, the control, their laws but also on their values at previous time instants. The dynamics of this work are close to those in the paper of Wang, Chen and Huang [19]. In [19], the adjoint equation is an anticipated backward stochastic differential equation (ABSDE) driven by both a $\mathrm{fBm}$ and a standard Brownian motion, and the integral with respect to the $\mathrm{fBm}$ is defined in the Stratonovich sense. Our work is also inspired by the recent paper of Buckdahn and Jing [4] where the system has a past-dependence feature. In [4] the dynamic of the adjoint process is driven by a standard Brownian motion; here the anticipated BSDE is driven by a fBM.

The approach used here is an extension of the work of Biagini, Hu, Øksendal and Sulem [2], where the dynamic of the system is of delayed mean-field type. In our paper, we establish necessary and sufficient maximum principles and we illustrate our study by solving two optimal control problems: a mean-field optimal consumption problem for cash flow with delay and a linear-quadratic (LQ) problem with delay.

We now present the general problem we consider more specifically:

\section{STATEMENT OF THE PROBLEM}

Let $B^{H}$ be a fractional Brownian motion on $\left(\Omega, \mathcal{F}, \mathbb{F}=\left(\mathcal{F}_{t}\right)_{t \geqslant 0}, \mathbb{P}\right)$ a filtered probability space. We consider the following mean-field controlled stochastic delay equation:

$$
\begin{aligned}
d X_{t} & =b\left(t, X_{t}, X_{t-\delta}, M_{t}, M_{t-\delta}, u_{t}\right) d t+\sigma\left(t, M_{t}, M_{t-\delta}\right) d B_{t}^{H}, \quad t \in[0, T], \\
X_{t} & =x_{0}(t), \quad t \in[-\delta, 0],
\end{aligned}
$$

where $M_{s}:=\mathbb{P}_{X_{s}}$ for $s \in[0, T]$ and $T, \delta>0$ are given constants. Here

$$
\begin{aligned}
& b: \Omega \times[0, T] \times \mathbb{R} \times \mathbb{R} \times \mathcal{P}_{2}(\mathbb{R}) \times \mathcal{P}_{2}(\mathbb{R}) \times \mathcal{U} \rightarrow \mathbb{R}, \\
& \sigma:[0, T] \times \mathcal{P}_{2}(\mathbb{R}) \times \mathcal{P}_{2}(\mathbb{R}) \rightarrow \mathbb{R}
\end{aligned}
$$


are given functions such that, for all $t \in[0, T], b(\cdot, t, x, \bar{x}, m, \bar{m}, u)$ is supposed to be $\mathcal{F}_{t}$-measurable for all $x, \bar{x} \in \mathbb{R}, u \in \mathcal{U}, m, \bar{m} \in \mathcal{P}_{2}(\mathbb{R})$. The function $\sigma$ is assumed to be deterministic and such that its integral with respect to the $\mathrm{fBm}$ is a Wiener type integral. $\mathcal{P}_{2}(\mathbb{R})$ denotes the space of all probability measures $m$ on $(\mathbb{R}, \mathcal{B}(\mathbb{R}))$ such that $\int_{\mathbb{R}}|x|^{2} m(d x)<+\infty$. The function $x_{0}$ is assumed to be continuous and deterministic. The set $\mathcal{U} \subset \mathbb{R}$ consists of all admissible control values. Let $\mathcal{F}$ denote the filtration generated by the fBm $B^{H}$ and let $\mathcal{G}_{t} \subseteq \mathcal{F}_{t}$ be a subfiltration of $\mathcal{F}$ representing the information available to the controller who takes the decision $u_{t}$ at time $t$. We also denote by $\mathcal{A}_{\mathcal{G}}$ a given family of admissible control processes required to be $\mathcal{U}$-valued and $\mathcal{G}$-adapted. Throughout the paper, we assume that $X$ exists and belongs to $L^{2}(\Omega \times[0, T])$. The performance functional is assumed to have the form

$$
J(u)=\mathbb{E}\left[g\left(X_{T}, M_{T}\right)+\int_{0}^{T} f\left(t, X_{t}, X_{t-\delta}, M_{t}, M_{t-\delta}, u_{t}\right) d t\right],
$$

where

$$
\begin{aligned}
& g: \Omega \times \mathbb{R} \times \mathcal{P}_{2}(\mathbb{R}) \rightarrow \mathbb{R}, \\
& f: \Omega \times[0, T] \times \mathbb{R} \times \mathbb{R} \times \mathcal{P}_{2}(\mathbb{R}) \times \mathcal{P}_{2}(\mathbb{R}) \times \mathcal{U} \rightarrow \mathbb{R}
\end{aligned}
$$

are given functions such that for all $t \in[0, T], f(\cdot, t, x, \bar{x}, m, \bar{m}, u)$ is assumed to be $\mathcal{F}_{t}$-measurable for all $x, \bar{x} \in \mathbb{R}, u \in \mathcal{U}, m, \bar{m} \in \mathcal{P}_{2}(\mathbb{R})$. The function $g(\cdot, x, m)$ is assumed to be $\mathcal{F}_{T}$-measurable for all $x \in \mathbb{R}$ and $m \in \mathcal{P}_{2}(\mathbb{R})$. We also assume the integrability condition

$$
\mathbb{E}\left[\left|g\left(X_{T}, M_{T}\right)\right|+\int_{0}^{T}\left|f\left(t, X_{t}, X_{t-\delta}, M_{t}, M_{t-\delta}, u_{t}\right)\right| d t\right]<+\infty .
$$

The functions $\sigma, b, f$ and $g$ are assumed to be continuously differentiable with respect to $x, \bar{x}, u$ with bounded derivatives and admit Fréchet bounded derivatives with respect to $m, \bar{m}$.

The problem we consider is the following:

Problem. Find a control $u^{*} \in \mathcal{A}_{\mathcal{G}}$ such that

$$
J\left(u^{*}\right)=\sup _{u \in \mathcal{A}_{\mathcal{G}}} J(u) .
$$

Any control $u^{*} \in \mathcal{A}_{\mathcal{G}}$ satisfying $(2.1)$ is called an optimal control.

\section{GENERALITIES}

In this section we give some preliminaries concerning fractional Brownian motion based on fractional white noise calculus and some generalities on differentiability with respect to measures. For more details about fractional white noise theory, the reader may consult the books [3], [12]. 
3.1. Fractional Brownian motion. Let $T>0$ be a finite time horizon and $\Omega$ the space $\mathcal{S}^{\prime}([0, T])$ of tempered distributions on $[0, T]$, which is the dual of the Schwartz space $\mathcal{S}([0, T])$ of rapidly decreasing smooth functions on $[0, T]$.

For $1 / 2<H<1$, we put

$$
\varphi_{H}(t, s)=H(2 H-1)|t-s|^{2 H-2}, \quad s, t \in[0, T] .
$$

If $\omega \in \mathcal{S}^{\prime}([0, T])$ and $f \in \mathcal{S}([0, T])$, we let $\langle\omega, f\rangle=\omega(f)$ denote the action of $\omega$ on $f$; it can be extended to $f:[0, T] \rightarrow \mathbb{R}$ such that

$$
\|f\|_{H}^{2}:=\int_{0}^{T} \int_{0}^{T} f(s) f(t) \varphi_{H}(t, s) d s d t<+\infty .
$$

The space of all such functions $f$ is denoted by $\mathbb{L}_{H}^{2}([0, T])$. Since the map $f \mapsto$ $e^{-\|f\|_{H}^{2} / 2}$ with $f \in \mathcal{S}([0, T])$ is positive definite on $\mathcal{S}([0, T])$, by the BochnerMinlos theorem there exists a probability measure $\mathbb{P}^{H}=\mathbb{P}$ on the Borel subsets $\mathcal{B}(\Omega)$ such that

$$
\int_{\Omega} e^{i\langle\omega, f\rangle} d \mathbb{P}(\omega)=e^{-\|f\|_{H}^{2} / 2}, \quad \forall f \in \mathcal{S}([0, T]) .
$$

It follows from (3.1) that

$$
\mathbb{E}[\langle\cdot, f\rangle]=0 \quad \text { and } \quad \mathbb{E}\left[\langle\cdot, f\rangle^{2}\right]=\|f\|_{H}^{2}
$$

where $\mathbb{E}$ denotes the expectation under the probability measure $\mathbb{P}$. Hence, if we put $B_{t}^{H}:=\left\langle\omega, \chi_{[0, t]}(\cdot)\right\rangle$, then using (3.2) we see that $B^{H}$ is a fractional Brownian motion with Hurst parameter $H$, that is, a centred Gaussian process with covariance function

$$
\mathbb{E}\left[B_{t}^{H} B_{s}^{H}\right]=\frac{1}{2}\left(t^{2 H}+s^{2 H}-|t-s|^{2 H}\right), \quad s, t \in[0, T] .
$$

From now on, we endow $\Omega$ with the natural filtration $\mathcal{F}:=\left\{\mathcal{F}_{t}\right\}_{t \in[0, T]}$ generated by $B^{H}$.

Lemma 3.1. If $f, g \in \mathbb{L}_{H}^{2}([0, T])$, then the Wiener integrals $\int_{0}^{T} f(s) d B^{H}(s)$ and $\int_{0}^{T} g(s) d B^{H}(s)$ are well defined as zero mean Gaussian random variables with variances $\|f\|_{H}^{2}$ and $\|g\|_{H}^{2}$ respectively and

$$
\mathbb{E}\left[\int_{0}^{T} f(s) d B^{H}(s) \int_{0}^{T} g(s) d B^{H}(s)\right]=\int_{0}^{T} \int_{0}^{T} f(s) g(t) \varphi_{H}(t, s) d t d s .
$$

We denote by $\tilde{\mathcal{S}}$ the set of all polynomial functions of $\int_{0}^{T} \psi_{j}(t) d B^{H}(t)$, denoted by $B^{H}\left(\psi_{j}\right)$. For $F \in \tilde{\mathcal{S}}$ having the form $F=g\left(B^{H}\left(\psi_{1}\right), \ldots, B^{H}\left(\psi_{n}\right)\right)$, 
where $g$ is a polynomial function of $n$ variables, we define its Malliavin derivative by $D_{s}^{H} F:=\sum_{i=1}^{n} \frac{\partial g}{\partial x_{i}}\left(B^{H}\left(\psi_{1}\right), \ldots, B^{H}\left(\psi_{n}\right)\right) \psi_{i}(s), 0 \leqslant s \leqslant T$. We also introduce another derivative $\mathbb{D}_{t}^{H} F:=\int_{0}^{T} D_{r}^{H} F \varphi_{H}(r, t) d r$. Let $\mathcal{L}_{H}^{1,2}([0, T])$ be the set of processes $G:[0, T] \times \Omega \rightarrow \mathbb{R}$ such that $\mathbb{D}_{s}^{H} G(s)$ exists for all $s \in[0, T]$ and

$$
\|G\|_{\mathcal{L}_{H}^{1,2}}^{2}:=\mathbb{E}\left[\int_{0}^{T} \int_{0}^{T} G(s) G(t) \varphi_{H}(t, s) d t d s+\left(\int_{0}^{T} \mathbb{D}_{s}^{H} G(s) d s\right)^{2}\right]<+\infty .
$$

The integral $\int_{0}^{T} G(s) d B^{H}(s)$ denotes the fractional Wick-Itô-Skorokhod (fWIS) integral of the process $G$ with respect to $B^{H}$. We recall its construction: if $G$ belongs to the family $\mathbb{S}$ of step functions of the form $G(t, \omega)=$ $\sum_{i=1}^{N} G_{i}(\omega) \chi_{\left[t_{i}, t_{i+1}[\right.}(t)$, where $0 \leqslant t_{1}<\cdots<t_{N+1} \leqslant T$, then the fWIS integral is defined naturally as $\int_{0}^{T} G(t, \omega) d B^{H}(t):=\sum_{i=1}^{N} G_{i}(\omega) \diamond\left(B^{H}\left(t_{i+1}\right)-\right.$ $\left.B^{H}\left(t_{i}\right)\right)$, where $\diamond$ denotes the Wick product (see [3] for its definition). For $G \in \mathbb{S} \cap \mathcal{L}_{H}^{1,2}([0, T])$, we have the isometry

$$
\mathbb{E}\left[\left(\int_{0}^{T} G(t) d B^{H}(t)\right)^{2}\right]=\mathbb{E}\left[\int_{0}^{T} \int_{0}^{T} G(s) G(t) \varphi_{H}(t, s) d t d s+\left(\int_{0}^{T} \mathbb{D}_{s}^{H} G(s) d s\right)^{2}\right] .
$$

Therefore, we can extend the integral $\int_{0}^{T} G(t) d B^{H}(t)$ to $\mathcal{L}_{H}^{1,2}([0, T])$. Note that if $G_{1}, G_{2} \in \mathcal{L}_{H}^{1,2}([0, T])$, by polarization we have

$$
\begin{aligned}
\mathbb{E}\left[\int_{0}^{T} G_{1}(t) d B^{H}(t) \int_{0}^{T} G_{2}(t) d B^{H}(t)\right]= & \mathbb{E}\left[\int_{0}^{T} G_{1}(s) G_{2}(t) \varphi_{H}(s, t) d s d t\right] \\
& +\mathbb{E}\left[\int_{0}^{T} \int_{0}^{T} \mathbb{D}_{s}^{H} G_{1}(s) \mathbb{D}_{t}^{H} G_{2}(t) d s d t\right] .
\end{aligned}
$$

This integral satisfies $\mathbb{E}\left[\int_{0}^{T} G(t) d B^{H}(t)\right]=0$ for all $G \in \mathcal{L}_{H}^{1,2}([0, T])$.

Proposition 3.1 (Integration by parts). Let $X$ and $Y$ be processes of the form

$$
\begin{array}{ll}
d X(t)=F_{1}(t) d t+G_{1}(t) d B^{H}(t), & X(0)=x \in \mathbb{R}, \\
d Y(t)=F_{2}(t) d t+G_{2}(t) d B^{H}(t), & Y(0)=y \in \mathbb{R},
\end{array}
$$

where $F_{1}:[0, T] \times \Omega \rightarrow \mathbb{R}, F_{2}:[0, T] \times \Omega \rightarrow \mathbb{R}, G_{1}:[0, T] \times \Omega \rightarrow \mathbb{R}$ and $G_{2}:[0, T] \times \Omega \rightarrow \mathbb{R}$ are given processes such that $G_{1}, G_{2} \in \mathcal{L}_{H}^{1,2}([0, T])$.

(1) Then, for $T>0$,

$$
\begin{aligned}
\mathbb{E} & {[X(T) Y(T)]=x y+\mathbb{E}\left[\int_{0}^{T} X(s) d Y(s)\right]+\mathbb{E}\left[\int_{0}^{T} Y(s) d X(s)\right] } \\
+ & \mathbb{E}\left[\int_{0}^{T} \int_{0}^{T} G_{1}(t) G_{2}(s) \varphi_{H}(t, s) d s d t\right]+\mathbb{E}\left[\int_{0}^{T} \int_{0}^{T} \mathbb{D}_{t}^{H} G_{1}(t) \mathbb{D}_{s}^{H} G_{2}(s) d s d t\right]
\end{aligned}
$$

provided that the first two integrals exist. 
(2) In particular, if $G_{1}$ or $G_{2}$ is deterministic, then

$$
\begin{aligned}
\mathbb{E}[X(T) Y(T)]= & x y+\mathbb{E}\left[\int_{0}^{T} X(s) d Y(s)\right] \\
& +\mathbb{E}\left[\int_{0}^{T} Y(s) d X(s)\right]+\mathbb{E}\left[\int_{0}^{T} \int_{0}^{T} G_{1}(t) G_{2}(s) \varphi_{H}(t, s) d s d t\right] .
\end{aligned}
$$

3.2. Differentiability of functions of measures. Let $\mathcal{P}(\mathbb{R})$ be the space of all probability measures on $(\mathbb{R}, \mathcal{B}(\mathbb{R}))$ and define

$$
\mathcal{P}_{p}(\mathbb{R})=\left\{m \in \mathcal{P}(\mathbb{R}): \int_{\mathbb{R}}|x|^{p} m(d x)<+\infty\right\} .
$$

- Differentiability of functions of measures: The notion of differentiability for functions of measures that we will use is the one introduced by Lions in his course at Collège de France [16] and summarized by Cardaliaguet [6]; we also refer to Carmona and Delarue [8]. This notion is based on lifting of functions $\mathcal{P}_{2}(\mathbb{R}) \ni$ $m \mapsto \sigma(m)$ to functions $L^{2}(\tilde{\Omega} ; \mathbb{R}) \ni \tilde{\xi} \mapsto \tilde{\sigma}(\tilde{\xi})$, over some probability space $(\tilde{\Omega}, \tilde{\mathcal{F}}, \tilde{\mathbb{P}})$, by setting $\tilde{\sigma}(\tilde{\xi}):=\sigma\left(\tilde{\mathbb{P}}_{\tilde{\xi}}\right)$.

Definition 3.1. A function $\sigma$ is said to be differentiable at $m_{0} \in \mathcal{P}_{2}(\mathbb{R})$ if there exists a random variable $\tilde{\xi}_{0} \in L^{2}(\tilde{\Omega}, \tilde{\mathcal{F}}, \tilde{\mathbb{P}})$ over some probability space $(\tilde{\Omega}, \tilde{\mathcal{F}}, \tilde{\mathbb{P}})$ with $\tilde{\mathbb{P}}_{\tilde{\xi}_{0}}=m_{0}$ such that $\tilde{\sigma}: L^{2}(\tilde{\Omega}, \tilde{\mathcal{F}}, \tilde{\mathbb{P}}) \rightarrow \mathbb{R}$ is Fréchet differentiable at $\tilde{\xi}_{0}$.

We suppose for simplicity that $\tilde{\sigma}: L^{2}(\tilde{\Omega}, \tilde{\mathcal{F}}, \tilde{\mathbb{P}}) \rightarrow \mathbb{R}$ is Fréchet differentiable. We denote its Fréchet derivative at $\tilde{\xi}_{0}$ by $D \tilde{\sigma}\left(\tilde{\xi}_{0}\right)$. Recall that $D \tilde{\sigma}\left(\tilde{\xi}_{0}\right)$ is a continuous linear mapping, i.e. $D \tilde{\sigma}\left(\tilde{\xi}_{0}\right) \in L\left(L^{2}(\tilde{\Omega}, \tilde{\mathcal{F}}, \tilde{\mathbb{P}}), \mathbb{R}\right)$. With the identification $L\left(L^{2}(\tilde{\Omega}, \tilde{\mathcal{F}}, \tilde{\mathbb{P}}), \mathbb{R}\right) \equiv L^{2}(\tilde{\Omega}, \tilde{\mathcal{F}}, \tilde{\mathbb{P}})$ given by the Riesz representation theorem, $D \tilde{\sigma}\left(\tilde{\xi}_{0}\right)$ is viewed as an element of $L^{2}(\tilde{\Omega}, \tilde{\mathcal{F}}, \tilde{\mathbb{P}})$, hence we can write

$$
\sigma(m)-\sigma\left(m_{0}\right)=\tilde{\sigma}(\tilde{\xi})-\tilde{\sigma}\left(\tilde{\xi}_{0}\right)=\tilde{\mathbb{E}}\left[(D \tilde{\sigma})\left(\tilde{\xi}_{0}\right) \cdot\left(\tilde{\xi}-\tilde{\xi}_{0}\right)\right]+o\left(\tilde{\mathbb{E}}\left[\left\|\tilde{\xi}-\tilde{\xi}_{0}\right\|_{L^{2}}\right]\right)
$$

as $\tilde{\mathbb{E}}\left[\left\|\tilde{\xi}-\tilde{\xi}_{0}\right\|_{L^{2}}\right] \rightarrow 0$, where $\tilde{\xi}$ is a random variable with law $m$. Moreover, according to Cardaliaguet [6], there exists a Borel function $h_{m_{0}}: \mathbb{R} \rightarrow \mathbb{R}$ such that $D \tilde{\sigma}\left(\tilde{\xi}_{0}\right)=h_{m_{0}}\left(\tilde{\xi}_{0}\right)$, $\tilde{\mathbb{P}}_{\text {-a.s. }}$. We define the derivative of $\sigma$ with respect to the measure $m$ at $m_{0}$ by putting $\partial_{m} \sigma\left(m_{0}\right)(x):=h_{m_{0}}(x)$. Notice that $\partial_{m} \sigma\left(m_{0}\right)(x)$ is defined $m_{0}(d x)$-a.e. uniquely. Therefore, the previous differentiation formula is invariant under modification of the space $\tilde{\Omega}$ where the random variables $\tilde{\xi}_{0}$ and $\tilde{\xi}$ are defined.

- Joint concavity: We will need the joint concavity of a function on the product $\mathbb{R} \times \mathcal{P}_{2}(\mathbb{R})$. A differentiable function $b$ defined on $\mathbb{R} \times \mathcal{P}_{2}(\mathbb{R})$ is concave if for every 
$\left(x^{\prime}, m^{\prime}\right),(x, m) \in \mathbb{R} \times \mathcal{P}_{2}(\mathbb{R})$, we have

$$
b\left(x^{\prime}, m^{\prime}\right)-b(x, m)-\partial_{x} b(x, m)\left(x^{\prime}-x\right)-\tilde{\mathbb{E}}\left[\partial_{m} b(x, m)(\tilde{X})\left(\tilde{X}^{\prime}-\tilde{X}\right)\right] \leqslant 0
$$

whenever $\tilde{X}, \tilde{X}^{\prime} \in L^{2}(\tilde{\Omega}, \tilde{\mathcal{F}}, \tilde{\mathbb{P}}, \mathbb{R})$ with laws $m$ and $m^{\prime}$ respectively.

\section{NECESSARY MAXIMUM PRINCIPLE}

In this section, we will establish a maximum principle of necessary type. Let $u^{*}$ be a control in $\mathcal{A}_{\mathcal{G}}$. We first compute the Gâteaux derivative of the performance functional $J$ at $u^{*}$ in all directions. In order to do so, we make the following assumption, for any bounded control $v$, there exists $\eta>0$ such that the control $u^{\theta}$ defined by $u^{\theta}:=u^{*}+\theta v$ belongs to $\mathcal{A}_{\mathcal{G}}$ for any $\theta \in(-\eta, \eta)$. We denote by $X^{\theta}:=X^{u^{\theta}}, X^{*}:=X^{u^{*}}$ the controlled state processes corresponding to $u^{\theta}$ and $u^{*}$ respectively.

For $u^{*} \in \mathcal{A}_{\mathcal{G}}$ and the associated controlled state process $X^{*}$, let $Y_{t}:=$ $\left.\frac{d}{d \theta} X_{t}^{\theta}\right|_{\theta=0}$. Hence $Y$ satisfies the following SDDE:

$$
\begin{aligned}
d Y_{t}= & \left\{\partial_{x} b^{*}(t) Y_{t}+\partial_{\bar{x}} b^{*}(t) Y_{t-\delta}+\tilde{\mathbb{E}}\left[\partial_{m} b^{*}(t)\left(\tilde{X}_{t}^{*}\right) \tilde{Y}_{t}\right]\right. \\
& \left.+\tilde{\mathbb{E}}\left[\partial_{\bar{m}} b^{*}(t)\left(\tilde{X}_{t-\delta}^{*}\right) \tilde{Y}_{t-\delta}\right]+\partial_{u} b^{*}(t) v(t)\right\} d t \\
& +\left\{\tilde{\mathbb{E}}\left[\partial_{m} \sigma^{*}(t)\left(\tilde{X}_{t}^{*}\right) \tilde{Y}_{t}\right]+\tilde{\mathbb{E}}\left[\partial_{\bar{m}} \sigma^{*}(t)\left(\tilde{X}_{t-\delta}^{*}\right) \tilde{Y}_{t-\delta}\right]\right\} d B_{t}^{H} \\
Y_{t}= & 0, \quad t \in[-\delta, 0],
\end{aligned}
$$

where we use the following notations:

$$
\begin{aligned}
\partial_{x} b^{*}(t) & :=\partial_{x} b\left(t, X_{t}^{*}, X_{t-\delta}^{*}, M_{t}^{*}, M_{t-\delta}^{*}, u_{t}^{*}\right), \\
\partial_{m} b^{*}(t)(\cdot) & :=\partial_{m} b\left(t, X_{t}^{*}, X_{t-\delta}^{*}, M_{t}^{*}, M_{t-\delta}^{*}, u_{t}^{*}\right)(\cdot), \\
\partial_{m} \sigma^{*}(t)(\cdot) & :=\partial_{m} \sigma\left(t, M_{t}^{*}, M_{t-\delta}^{*}\right)(\cdot) ;
\end{aligned}
$$

moreover, $(\tilde{X}, \tilde{Y}, \tilde{u})$ is an independent copy of $(X, Y, u)$ defined on some probability space $(\tilde{\Omega}, \tilde{\mathcal{F}}, \tilde{\mathbb{P}})$ and $\tilde{\mathbb{E}}$ denotes the expectation on $(\tilde{\Omega}, \tilde{\mathcal{F}}, \tilde{\mathbb{P}})$.

REMARK 4.1. From the definition of the tilde random variables and since $\sigma$ is deterministic, we have $\tilde{\mathbb{E}}\left[\partial_{m} \sigma^{*}(t)\left(\tilde{X}_{t}^{*}\right) \tilde{Y}_{t}\right]=\mathbb{E}\left[\partial_{m} \sigma^{*}(t)\left(X_{t}^{*}\right) Y_{t}\right]$. Note that in the previous notations, $\tilde{\mathbb{E}}\left[\partial_{m} b^{*}(t)\left(\tilde{X}_{t}^{*}\right) \tilde{Y}_{t}\right]$ is a function of the random vector $\left(X_{t}^{*}, X_{t-\delta}^{*}, u_{t}^{*}\right)$ as it stands for

$$
\left.\tilde{\mathbb{E}}\left[\partial_{m} b\left(t, x, \bar{x}, M_{t}^{*}, M_{t-\delta}^{*}, u\right)\left(\tilde{X}_{t}^{*}\right) \tilde{Y}_{t}\right]\right|_{x=X_{t}^{*}, \bar{x}=X_{t-\delta}^{*}, u=u_{t}^{*}} .
$$

We assume that the derivative process $Y$ exists and belongs to $L^{2}(\Omega \times[0, T])$ and that $\psi_{\delta}^{*}: t \mapsto \tilde{\mathbb{E}}\left[\partial_{m} \sigma^{*}(t)\left(\tilde{X}^{*}(t)\right) \tilde{Y}(t)\right]+\tilde{\mathbb{E}}\left[\partial_{\bar{m}} \sigma^{*}(t)\left(\tilde{X}^{*}(t-\delta)\right) \tilde{Y}(t-\delta)\right]$ is in $\mathbb{L}_{H}^{2}([0, T])$, the integral with respect to the $\mathrm{fBm}$ is therefore well defined in the 
Wiener sense. We compute $\left.\frac{d}{d \theta} J\left(u^{\theta}\right)\right|_{\theta=0}$ using the expression of the performance functional to get

$$
\begin{aligned}
& \text { 4.2) }\left.\quad \frac{d}{d \theta} J\left(u^{\theta}\right)\right|_{\theta=0} \\
& =\mathbb{E}\left[\partial_{x} g^{*}(T) Y_{T}+\tilde{\mathbb{E}}\left[\partial_{m} g^{*}(T)\left(\tilde{X}_{T}^{*}\right) \tilde{Y}_{T}\right]\right]+\mathbb{E}\left[\int_{0}^{T}\left\{\partial_{x} f^{*}(t) Y_{t}+\partial_{\bar{x}} f^{*}(t) Y_{t-\delta}\right\} d t\right] \\
& +\mathbb{E}\left[\int_{0}^{T}\left\{\tilde{\mathbb{E}}\left[\partial_{m} f^{*}(t)\left(\tilde{X}_{t}^{*}\right) \tilde{Y}_{t}\right]+\tilde{\mathbb{E}}\left[\partial_{\bar{m}} f^{*}(t)\left(\tilde{X}_{t-\delta}^{*}\right) \tilde{Y}_{t-\delta}\right]+\partial_{u} f^{*}(t) v_{t}\right\} d t\right]
\end{aligned}
$$

where we have used the simplified notations

$$
\begin{aligned}
\partial_{x} g^{*}(T) & :=\partial_{x} g\left(X_{T}^{*}, M_{T}^{*}\right), \\
\partial_{m} g^{*}(T)(\cdot) & :=\partial_{m} g\left(X_{T}^{*}, M_{T}^{*}\right)(\cdot), \\
\partial_{x} f^{*}(t) & :=\partial_{x} f\left(t, X_{t}^{*}, X_{t-\delta}^{*}, M_{t}^{*}, M_{t-\delta}^{*}, u_{t}^{*}\right), \\
\partial_{m} f^{*}(t)(\cdot) & :=\partial_{m} f\left(t, X_{t}^{*}, X_{t-\delta}^{*}, M_{t}^{*}, M_{t-\delta}^{*}, u_{t}^{*}\right)(\cdot), \\
\partial_{m} \sigma^{*}(t)(\cdot) & :=\partial_{m} \sigma\left(t, M_{t}^{*}, M_{t-\delta}^{*}\right)(\cdot) .
\end{aligned}
$$

In order to determine the adjoint backward equation associated to $\left(u^{*}, X^{*}\right)$, we suppose that it has the form

$$
\left\{\begin{array}{l}
d p_{t}^{*}=-\mathbb{E}\left[\alpha(t) \mid \mathcal{F}_{t}\right] d t+q_{t}^{*} d B_{t}^{H}, \quad t \in[0, T], \\
p_{T}^{*}=\partial_{x} g^{*}(T)+\tilde{\mathbb{E}}\left[\partial_{m} \tilde{g}^{*}(T)\left(X_{T}\right)\right]
\end{array}\right.
$$

where $\left(p^{*}, q^{*}\right)$ is assumed to be in $\mathcal{L}_{H}^{1,2}([0, T]) \times \mathcal{L}_{H}^{1,2}([0, T])$, the integral with respect to the fBm is a fractional Wick-Itô-Skorokhod integral and $\alpha$ is a stochastic process which we aim to determine.

Applying the integration by parts formula of Proposition 3.1 to $p_{t}^{*}$ and $Y_{t}$, we obtain

$$
\begin{aligned}
\mathbb{E}\left[p_{T}^{*} Y_{T}\right]= & \mathbb{E}\left[\int_{0}^{T} p_{t}^{*} d Y_{t}\right]+\mathbb{E}\left[\int_{0}^{T} Y_{t} d p_{t}^{*}\right]+\mathbb{E}\left[\int_{0}^{T} \int_{0}^{T} q_{t}^{*} \psi_{\delta}^{*}(t) \varphi_{H}(s, t) d s d t\right] \\
= & \mathbb{E}\left[\int_{0}^{T} p_{t}^{*}\left\{\partial_{x} b^{*}(t) Y_{t}+\partial_{\bar{x}} b^{*}(t) Y_{t-\delta}\right)+\tilde{\mathbb{E}}\left[\partial_{m} b^{*}(t)\left(\tilde{X}_{t}^{*}\right) \tilde{Y}_{t}\right]\right. \\
& \left.\left.+\tilde{\mathbb{E}}\left[\partial_{\bar{m}} b^{*}(t)\left(\tilde{X}_{t-\delta}^{*}\right) \tilde{Y}_{t-\delta}\right]+\partial_{u} b^{*}(t) v_{t}\right\} d t\right]-\mathbb{E}\left[\int_{0}^{T} Y_{t} \alpha(t) d t\right] \\
& +\mathbb{E}\left[\int_{0}^{T} \int_{0}^{T} q_{s}^{*} \psi_{\delta}^{*}(t) \varphi_{H}(s, t) d s d t\right]
\end{aligned}
$$


where we assume that $Y(t) q^{*}(t) \in \mathcal{L}_{H}^{1,2}([0, T])$ and $\psi_{\delta}^{*}(t) p^{*}(t) \in \mathcal{L}_{H}^{1,2}([0, T])$. By Fubini's theorem and Remark 4.1 , replacing $\psi_{\delta}^{*}(t)$ by its value and applying a change of variables using the fact that $Y(t)=0$ for all $t \in[-\delta, 0]$, we get

$$
\begin{aligned}
& \mathbb{E}\left[p_{T}^{*} Y_{T}\right] \\
= & \mathbb{E}\left[\int _ { 0 } ^ { T } Y _ { t } \left\{p_{t}^{*} \partial_{x} b^{*}(t)+p_{t+\delta}^{*} \partial_{\bar{x}} b^{*}(t+\delta) \chi_{[0, T-\delta]}(t)-\alpha(t)+\tilde{\mathbb{E}}\left[\tilde{p}_{t}^{*} \partial_{m} \tilde{b}^{*}(t)\left(X_{t}^{*}\right)\right]\right.\right. \\
+ & \tilde{\mathbb{E}}\left[\tilde{p}^{*}(t+\delta) \partial_{\bar{m}} \tilde{b}^{*}(t+\delta)\left(X_{t}^{*}\right)\right] \chi_{[0, T-\delta]}(t)+\int_{0}^{T} \tilde{\mathbb{E}}\left[\tilde{q}^{*}(s)\right]\left\{\partial_{m} \sigma^{*}(t)\left(X_{t}^{*}\right) \varphi_{H}(t, s)\right. \\
+ & \left.\left.\left.\partial_{\bar{m}} \sigma^{*}(t+\delta)\left(X_{t}^{*}\right) \chi_{[0, T-\delta]}(t) \varphi_{H}(t+\delta, s)\right\} d s\right\} d t\right]+\mathbb{E}\left[\int_{0}^{T} p_{t}^{*} \partial_{u} b^{*}(t) v_{t} d t\right],
\end{aligned}
$$

where $\left(\tilde{p}^{*}, \tilde{q}^{*}\right)$ is an independent copy of $\left(p^{*}, q^{*}\right)$ defined on some probability space $(\tilde{\Omega}, \tilde{\mathcal{F}}, \tilde{\mathbb{P}})$. On the other hand, if we assume that $u^{*}$ is an optimal control then by substituting (4.4) in (4.2) and using the terminal value of the BSDE, we get

$$
\begin{aligned}
& \mathbb{E}\left[\int _ { 0 } ^ { T } Y _ { t } \left\{p_{t}^{*} \partial_{x} b^{*}(t)+p_{t+\delta}^{*} \partial_{\bar{x}} b^{*}(t+\delta) \chi_{[0, T-\delta]}(t)+\tilde{\mathbb{E}}\left[\tilde{p}_{t}^{*} \partial_{m} \tilde{b}^{*}(t)\left(X_{t}^{*}\right)\right]\right.\right. \\
& \quad+\tilde{\mathbb{E}}\left[\tilde{p}_{t+\delta}^{*} \partial_{\bar{m}} \tilde{b}^{*}(t+\delta)\left(X_{t}^{*}\right)\right] \chi_{[0, T-\delta]}(t)+\int_{0}^{T} \tilde{\mathbb{E}}\left[\tilde{q}^{*}(s)\right]\left\{\partial_{m} \sigma^{*}(t)\left(X_{t}^{*}\right) \varphi_{H}(t, s)\right. \\
& \left.\quad+\partial_{\bar{m}} \sigma^{*}(t+\delta)\left(X_{t}^{*}\right) \chi_{[0, T-\delta]}(t) \varphi_{H}(t+\delta, s)\right\} d s-\alpha(t) \\
& \quad+\partial_{x} f^{*}(t)+\partial_{\bar{x}} f^{*}(t+\delta) \chi_{[0, T-\delta]}(t)+\tilde{\mathbb{E}}\left[\partial_{m} \tilde{f}^{*}(t)\left(X_{t}^{*}\right)\right] \\
& \left.\left.\quad+\tilde{\mathbb{E}}\left[\partial_{\bar{m}} \tilde{f}^{*}(t+\delta)\left(X_{t}^{*}\right)\right] \chi_{[0, T-\delta]}(t)\right\} d t\right]+\mathbb{E}\left[\int_{0}^{T}\left\{p_{t}^{*} \partial_{u} b^{*}(t)+\partial_{u} f^{*}(t)\right\} v_{t} d t\right] \leqslant 0 .
\end{aligned}
$$

Letting the integrand which contains $Y_{t}$ be zero, we get

$$
\begin{aligned}
& \alpha(t)=p_{t}^{*} \partial_{x} b^{*}(t)+p_{t+\delta}^{*} \partial_{\bar{x}} b^{*}(t+\delta) \chi_{[0, T-\delta]}(t)+\tilde{\mathbb{E}}\left[\tilde{p}_{t}^{*} \partial_{m} \tilde{b}^{*}(t)\left(X_{t}^{*}\right)\right] \\
& +\tilde{\mathbb{E}}\left[\tilde{p}_{t+\delta}^{*} \partial_{\bar{m}} \tilde{b}^{*}(t+\delta)\left(X_{t}^{*}\right)\right] \chi_{[0, T-\delta]}(t)+\int_{0}^{T} \tilde{\mathbb{E}}\left[\tilde{q}^{*}(s)\right]\left\{\partial_{m} \sigma^{*}(t)\left(X_{t}^{*}\right) \varphi_{H}(t, s)\right. \\
& \left.\quad+\partial_{\bar{m}} \sigma^{*}(t+\delta)\left(X_{t}^{*}\right) \chi_{[0, T-\delta]}(t) \varphi_{H}(t+\delta, s)\right\} d s+\partial_{x} f^{*}(t) \\
& \quad+\partial_{\bar{x}} f^{*}(t+\delta) \chi_{[0, T-\delta]}(t)+\tilde{\mathbb{E}}\left[\partial_{m} \tilde{f}^{*}(t)\left(X_{t}^{*}\right)\right]+\tilde{\mathbb{E}}\left[\partial_{\bar{m}} \tilde{f}^{*}(t+\delta)\left(X_{t}^{*}\right)\right] \chi_{[0, T-\delta]}(t),
\end{aligned}
$$

where, for simplicity of notation, we have set

$$
\begin{aligned}
\partial_{x} \tilde{b}^{*}(t) & :=\partial_{x} b\left(t, \tilde{X}_{t}^{*}, \tilde{X}_{t-\delta}^{*}, M_{t}^{*}, M_{t-\delta}^{*}, \tilde{u}_{t}^{*}\right), \\
\partial_{m} \tilde{b}^{*}(t)(\cdot) & :=\partial_{m} b\left(t, \tilde{X}_{t}^{*}, \tilde{X}_{t-\delta}^{*}, M_{t}^{*}, M_{t-\delta}^{*}, \tilde{u}_{t}^{*}\right)(\cdot), \\
\partial_{m} \tilde{f}^{*}(t)(\cdot) & :=\partial_{m} f\left(t, \tilde{X}_{t}^{*}, \tilde{X}_{t-\delta}^{*}, M_{t}^{*}, M_{t-\delta}^{*}, \tilde{u}_{t}^{*}\right)(\cdot) .
\end{aligned}
$$


We now define the Hamiltonian

$$
H: \Omega \times[0, T] \times \mathbb{R} \times \mathbb{R} \times \mathcal{P}_{2}(\mathbb{R}) \times \mathcal{P}_{2}(\mathbb{R}) \times \mathcal{U} \times \mathbb{R} \times \mathcal{R} \rightarrow \mathbb{R}
$$

associated to our problem by

$$
\begin{aligned}
& H(t, x, \bar{x}, m, \bar{m}, u, p, q(\cdot)) \\
= & f(t, x, \bar{x}, m, \bar{m}, u)+p b(t, x, \bar{x}, m, \bar{m}, u)+\sigma(t, m, \bar{m}) \int_{0}^{T} q(s) \varphi_{H}(s, t) d s,
\end{aligned}
$$

where $\mathcal{R}$ is the set of continuous functions from $[0, T]$ into $\mathbb{R}$.

For $u \in \mathcal{A}_{\mathbb{F}}$ with the corresponding solution $X=X^{u}$, define, whenever solutions exist, $(p, q):=\left(p^{u}, q^{u}\right)$, by the adjoint equation, in terms of the Hamiltonian, as follows:

$$
\left\{\begin{aligned}
d p_{t}= & -\left\{\partial_{x} H(t)+\mathbb{E}\left[\partial_{\bar{x}} H(t+\delta) \chi_{[0, T-\delta]}(t) \mid \mathcal{F}_{t}\right]+\tilde{\mathbb{E}}\left[\partial_{m} \tilde{H}(t)\left(X_{t}\right)\right]\right. \\
& \left.+\mathbb{E}\left[\tilde{\mathbb{E}}\left[\partial_{\bar{m}} \tilde{H}(t+\delta)\left(X_{t}\right) \chi_{[0, T-\delta]}(t)\right] \mid \mathcal{F}_{t}\right]\right\} d t+q_{t} d B_{t}^{H}, \quad t \in[0, T], \\
p_{T}= & \partial_{x} g(T)+\tilde{\mathbb{E}}\left[\partial_{m} \tilde{g}(T)\left(X_{T}\right)\right] .
\end{aligned}\right.
$$

We assume that $(p, q)$ is in $\mathcal{L}_{H}^{1,2}([0, T]) \times \mathcal{L}_{H}^{1,2}([0, T])$, and the integral with respect to the $\mathrm{fBm}$ is understood in the fractional Wick-Itô-Skorokhod sense.

For simplicity of notation, we have put

$$
\begin{aligned}
\partial_{x} H(t) & :=\partial_{x} H\left(t, X_{t}, X_{t-\delta}, M_{t}, M_{t-\delta}, u(t), p_{t}, q(\cdot)\right), \\
\partial_{m} \tilde{H}(t)(\cdot) & :=\partial_{m} H\left(t, \tilde{X}_{t}, \tilde{X}_{t-\delta}, M(t), M_{t-\delta}, \tilde{u}_{t}, \tilde{p}_{t}, \tilde{q}(\cdot)\right)(\cdot), \\
\partial_{x} g(T) & :=\partial_{x} g\left(X_{T}, M_{T}\right), \quad \partial_{m} \tilde{g}(T)(\cdot)=\partial_{m} g\left(\tilde{X}_{T}, M_{T}\right)(\cdot) .
\end{aligned}
$$

REMARK 4.2. By the definition of the Hamiltonian above, the time advanced BSDE 4.6 has a first part which is linear and a second part which contains the laws of the solution processes. This type of Backward Stochastic Differential Equations has never been studied before. However, when there is no mean-field terms and no advance in time, several resolutions were proposed: see for instance [2], [14] and for a more general setting [13]. In the section devoted to applications, we suggest some simplified mean-field dynamics where the resolution of the BSDE 4.6) is possible through recursive procedures.

In the following theorem we establish a necessary condition of optimality.

THEOREM 4.1 (Necessary condition of optimality). Assume that $u^{*} \in \mathcal{A}_{\mathcal{G}}$ with the corresponding controlled process $X^{*}$. Suppose that there exist solutions $p_{t}^{*}, q_{t}^{*}$ of the adjoint equation (4.6) associated to the pair $\left(u^{*}, X^{*}\right)$ and that the following assumptions are satisfied: 
- $Y_{t} q_{t}^{*} \in \mathcal{L}_{H}^{1,2}([0, T])$ and $\psi_{\delta}^{*}(t) p_{t}^{*} \in \mathcal{L}_{H}^{1,2}([0, T])$.

- For $s_{0} \in[0, T]$, and all bounded and $\mathcal{G}_{s_{0}}$-measurable random variables $\xi$, the control process $v$ defined by

$$
v_{t}:=\xi \chi_{\left[s_{0}, T\right]}(t), \quad t \in[0, T],
$$

belongs to $\mathcal{A}_{\mathcal{G}}$.

Then the following assertions are equivalent:

(1) For all bounded $v \in \mathcal{A}_{G},\left.\frac{d}{d \theta} J\left(u^{*}+\theta v\right)\right|_{\theta=0}=0$.

(2) For all $t \in[0, T], \mathbb{E}\left[\partial_{u} H^{*}(t) \mid \mathcal{G}_{t}\right]=0$ a.s.

Proof. Suppose that assertion (1) holds. Then we have

$$
\begin{aligned}
\frac{d}{d \theta} J\left(u^{*}+\theta v\right) & \left.\right|_{\theta=0} \\
= & \mathbb{E}\left[p_{T}^{*} Y_{T}\right]+\mathbb{E}\left[\int _ { 0 } ^ { T } \left\{\partial_{x} f^{*}(t) Y_{t}+\partial_{\bar{x}} f^{*}(t) Y_{t-\delta}\right.\right. \\
& \left.\left.+\tilde{\mathbb{E}}\left[\partial_{m} f^{*}(t)\left(\tilde{X}_{t}^{*}\right) \tilde{Y}_{t}\right]+\tilde{\mathbb{E}}\left[\partial_{\bar{m}} f^{*}(t)\left(\tilde{X}_{t-\delta}^{*}\right) \tilde{Y}_{t-\delta}\right]+\partial_{u} f^{*}(t) v_{t}\right\} d t\right] .
\end{aligned}
$$

Applying again the integration by parts formula of Proposition 3.1 to $p_{t}^{*}$ and $Y_{t}$, then using Fubini's theorem, replacing $\psi_{\delta}^{*}(t)$ by its value, and using a change of variable and the fact that $Y_{t}=0$ for all $t \in[-\delta, 0]$, we get

$$
\mathbb{E}\left[A_{1}\right]=\mathbb{E}\left[\int_{0}^{T} p_{t}^{*} d Y_{t}\right]+\mathbb{E}\left[\int_{0}^{T} Y_{t} d p_{t}^{*}\right]+\mathbb{E}\left[\int_{0}^{T} \int_{0}^{T} q^{*}(s) \psi_{\delta}^{*}(t) \varphi_{H}(t, s) d s d t\right]
$$

$$
\begin{aligned}
= & \mathbb{E}\left[\int _ { 0 } ^ { T } Y _ { t } \left\{p_{t}^{*} \partial_{x} b^{*}(t)+p_{t+\delta}^{*} \partial_{\bar{x}} b^{*}(t+\delta) \chi_{[0, T-\delta]}(t)\right.\right. \\
& +\tilde{\mathbb{E}}\left[\tilde{p}_{t}^{*} \partial_{m} \tilde{b}^{*}(t)\left(X_{t}^{*}\right)\right]+\tilde{\mathbb{E}}\left[\tilde{p}_{t+\delta}^{*} \partial_{\bar{m}} \tilde{b}^{*}(t+\delta)\left(X_{t}^{*}\right)\right] \chi_{[0, T-\delta]}(t) \\
& -\left\{\partial_{x} H^{*}(t)+\mathbb{E}\left[\partial_{\bar{x}} H^{*}(t+\delta) \chi_{[0, T-\delta]}(t) \mid \mathcal{F}_{t}\right]+\tilde{\mathbb{E}}\left[\partial_{m} \tilde{H}^{*}(t)\left(X_{t}^{*}\right)\right]\right. \\
& \left.+\mathbb{E}\left[\tilde{\mathbb{E}}\left[\partial_{\bar{m}} \tilde{H}^{*}(t+\delta)\left(X_{t}^{*}\right) \chi_{[0, T-\delta]}(t)\right] \mid \mathcal{F}_{t}\right]\right\} \\
& +\int_{0}^{T} \tilde{\mathbb{E}}\left[\tilde{q}^{*}(s)\right]\left\{\partial_{m} \sigma^{*}(t)\left(X_{t}^{*}\right) \varphi_{H}(t, s)\right. \\
& \left.\left.\left.+\partial_{\bar{m}} \sigma^{*}(t+\delta)\left(X_{t}^{*}\right) \chi_{[0, T-\delta]}(t) \varphi_{H}(t+\delta, s)\right\} d s\right\} d t\right]+\mathbb{E}\left[\int_{0}^{T} p_{t}^{*} \partial_{u} b^{*}(t) v_{t} d t\right],
\end{aligned}
$$

where $A_{1}:=\mathbb{E}\left[p^{*}(T) Y(T)\right]$. Then by (4.8), (4.9) and applying the definition of 
the Hamiltonian, we get

$$
\mathbb{E}\left[\int_{0}^{T} \partial_{u} H^{*}(t) v(t) d t\right]=0 .
$$

If we apply 4.10) to $v(t)=\xi \chi_{[s, T]}(t)$, where $\xi$ is bounded and $G_{s_{0}}$-measurable, $s \geqslant s_{0}$, we obtain

$$
\mathbb{E}\left[\int_{s}^{T} \partial_{u} H^{*}(t) \xi d t\right]=0 .
$$

Differentiating with respect to $s$, we obtain

$$
\mathbb{E}\left[\partial_{u} H^{*}(s) \xi\right]=0
$$

Since this holds for all $s \geqslant s_{0}$ and all $\xi$, we consequently have

$$
\mathbb{E}\left[\partial_{u} H^{*}\left(s_{0}\right) \mid \mathcal{G}_{s_{0}}\right]=0 .
$$

This shows that assertion (1) implies (2); and we can prove that (2) implies (1) by reversing the argument above since every bounded $v \in \mathcal{A}_{G}$ can be approximated by linear combinations of controls of the form (4.7).

\section{SUFFICIENT MAXIMUM PRINCIPLE}

In this section, we prove a sufficient stochastic maximum principle.

THEOREM 5.1 (Sufficient condition of optimality). Let $u^{*} \in \mathcal{A}_{\mathcal{G}}$ with corresponding controlled state $X^{*}$. Suppose that there exist solutions $p_{t}^{*}$, $q_{t}^{*}$ of the associated adjoint equation (4.6). Assume the following:

(1) $X_{t}^{u} q_{t}^{*} \in \mathcal{L}_{H}^{1,2}([0, T]), p_{t}^{*} \sigma\left(t, M_{t}, M_{t-\delta}\right) \in \mathcal{L}_{H}^{1,2}([0, T])$ for all $u \in \mathcal{A}_{\mathcal{G}}$.

(2) (Concavity) The functions

$$
(x, \bar{x}, m, \bar{m}, u) \mapsto H\left(t, x, \bar{x}, m, \bar{m}, u, p_{t}^{*}, q^{*}(\cdot)\right), \quad(x, m) \mapsto g(x, m),
$$

are concave for each $t \in[0, T]$ almost surely.

(3) (Maximum condition)

$$
\begin{aligned}
\mathbb{E}\left[H \left(t, X_{t}^{*}, X_{t-\delta}^{*},\right.\right. & \left.\left.M_{t}^{*}, M_{t-\delta}^{*}, u_{t}^{*}, p_{t}^{*}, q^{*}(\cdot)\right) \mid \mathcal{G}_{t}\right] \\
& =\sup _{u \in \mathcal{U}} \mathbb{E}\left[H\left(t, X_{t}^{*}, X_{t-\delta}^{*}, M_{t}^{*}, M_{t-\delta}^{*}, u, p_{t}^{*}, q^{*}(\cdot)\right) \mid \mathcal{G}_{t}\right]
\end{aligned}
$$

for all $t \in[0, T]$ almost surely.

Then $\left(u^{*}, X^{*}\right)$ is an optimal couple for our problem. 
Proof. Let $u \in \mathcal{A}_{\mathcal{G}}$ be a generic admissible control, and $X=X^{u}$ the corresponding controlled state process. By the definition of the performance functional $J$ given by (2), we have

$$
J(u)-J\left(u^{*}\right)=A_{2}+A_{3},
$$

where

$$
A_{2}:=\mathbb{E}\left[\int_{0}^{T}\left\{f(t)-f^{*}(t)\right\} d t\right], \quad A_{3}:=\mathbb{E}\left[g(T)-g^{*}(T)\right] .
$$

Applying the definition of the Hamiltonian, we get

$$
A_{2}=\mathbb{E}\left[\int_{0}^{T}\left\{H(t)-H^{*}(t)-p_{t}^{*} b^{\prime}(t)-\int_{0}^{T} q_{s}^{*} \sigma^{\prime}(t) \varphi_{H}(s, t) d s\right\} d t\right],
$$

where we use the following notations:

$$
\begin{aligned}
b(t) & :=b\left(t, X_{t}, X_{t-\delta}, M_{t}, M_{t-\delta}, u_{t}\right), \\
b^{*}(t) & :=b\left(t, X_{t}^{*}, X_{t-\delta}^{*}, M_{t}^{*}, M_{t-\delta}^{*}, u_{t}^{*}\right), \\
f(t) & :=f\left(t, X_{t}, X_{t-\delta}, M_{t}, M_{t-\delta}, u_{t}\right), \\
f^{*}(t) & :=f\left(t, X_{t}^{*}, X_{t-\delta}^{*}, M_{t}^{*}, M_{t-\delta}^{*}, u_{t}^{*}\right), \\
g(T) & :=g\left(X_{T}, M_{T}\right), \quad g^{*}(T):=g\left(X_{T}^{*}, M_{T}^{*}\right), \\
\sigma(t) & :=\sigma\left(t, M_{t}, M_{t-\delta}\right), \quad \sigma^{*}(t):=\sigma\left(t, M_{t}^{*}, M_{t-\delta}^{*}\right), \\
H(t) & :=H\left(t, X_{t}, X_{t-\delta}, M_{t}, M_{t-\delta}, u_{t}, p_{t}^{*}, q^{*}(\cdot)\right), \\
H^{*}(t) & :=H\left(t, X_{t}^{*}, X_{t-\delta}^{*}, M_{t}^{*}, M_{t-\delta}^{*}, u_{t}^{*}, p_{t}^{*}, q^{*}(\cdot)\right), \\
b^{\prime}(t) & :=b(t)-b^{*}(t), \quad \sigma^{\prime}(t):=\sigma(t)-\sigma^{*}(t), \quad X_{t}^{\prime}:=X_{t}-X_{t}^{*} .
\end{aligned}
$$

Now using the concavity of $g$ and the terminal value of the BSDE (4.6) associated to $\left(u^{*}, X^{*}\right)$, by Fubini's theorem we get

$$
\begin{aligned}
A_{3} & \leqslant \mathbb{E}\left[\partial_{x} g^{*}(T) X_{T}^{\prime}\right]+\mathbb{E}\left[\tilde{\mathbb{E}}\left[\partial_{m} g^{*}(T)\left(\tilde{X}_{T}\right) \tilde{X}_{T}^{\prime}\right]\right] \\
& =\mathbb{E}\left[\left(\partial_{x} g^{*}(T)+\tilde{\mathbb{E}}\left[\partial_{m} \tilde{g}^{*}(T)\left(X_{T}\right)\right]\right) X_{T}^{\prime}\right]=\mathbb{E}\left[p_{T}^{*} X_{T}^{\prime}\right] .
\end{aligned}
$$

Applying the integration by parts formula to $p_{t}^{*}$ and $X_{t}^{\prime}$, we get

$$
\begin{aligned}
\mathbb{E}\left[p_{T}^{*} X_{T}^{\prime}\right]= & \mathbb{E}\left[\int_{0}^{T} p_{t}^{*} d X_{t}^{\prime}\right]+\mathbb{E}\left[\int_{0}^{T} X_{t}^{\prime} d p_{t}^{*}\right]+\mathbb{E}\left[\int_{0}^{T} \int_{0}^{T} q_{s}^{*} \sigma^{\prime}(t) \varphi_{H}(t, s) d s d t\right] \\
= & \mathbb{E}\left[\int_{0}^{T} p_{t}^{*} b^{\prime}(t) d t\right]-\mathbb{E}\left[\int _ { 0 } ^ { T } X _ { t } ^ { \prime } \left\{\partial_{x} H^{*}(t)+\partial_{\bar{x}} H^{*}(t+\delta) \chi_{[0, T-\delta]}(t)\right.\right. \\
& \left.\left.+\tilde{\mathbb{E}}\left[\partial_{m} \tilde{H}^{*}(t)\left(X_{t}^{*}\right)\right]+\tilde{\mathbb{E}}\left[\partial_{\bar{m}} \tilde{H}^{*}(t+\delta)\left(X_{t}^{*}\right)\right] \chi_{[0, T-\delta]}(t)\right\} d t\right] \\
& +\mathbb{E}\left[\int_{0}^{T} \int_{0}^{T} q_{s}^{*} \sigma^{\prime}(t) \varphi_{H}(t, s) d t d s\right] .
\end{aligned}
$$


Note that by the change of variables $r=t+\delta$, we have

$\mathbb{E}\left[\int_{0}^{T-\delta} X_{t}^{\prime} \partial_{\bar{x}} H^{*}(t+\delta) d t\right]=\mathbb{E}\left[\int_{\delta}^{T} X_{r-\delta}^{\prime} \partial_{\bar{x}} H^{*}(r) d r\right]=\mathbb{E}\left[\int_{0}^{T} X_{r-\delta}^{\prime} \partial_{\bar{x}} H^{*}(r) d r\right]$,

where we use $\mathbb{E}\left[\int_{0}^{\delta} X_{r-\delta}^{\prime} \partial_{\bar{x}} H^{*}(r) d r\right]=\mathbb{E}\left[\int_{-\delta}^{0} X^{\prime}(u) \partial_{\bar{x}} H^{*}(u+\delta) d u\right]=0$ since $X_{u}^{\prime}=0$ for all $u \in[-\delta, 0]$, because $X_{t}^{*}=X_{t}=x_{0}(t)$ for all $t \in[-\delta, 0]$. Similarly, using the previous argument and Fubini's theorem we get

$\mathbb{E}\left[\int_{0}^{T} X_{t}^{\prime} \tilde{\mathbb{E}}\left[\partial_{\bar{m}} \tilde{H}^{*}(t+\delta)\left(X_{t}^{*}\right)\right] \chi_{[0, T-\delta]}(t) d t\right]=\mathbb{E}\left[\int_{0}^{T} \tilde{\mathbb{E}}\left[\partial_{\bar{m}} H^{*}(t)\left(\tilde{X}_{t-\delta}^{*}\right) \tilde{X}_{t-\delta}^{\prime}\right] d t\right]$.

Hence,

$$
\begin{aligned}
\mathbb{E}\left[p_{T}^{*} X_{T}^{\prime}\right] & \\
= & \mathbb{E}\left[\int_{0}^{T} p_{t}^{*} b^{\prime}(t) d t\right]-\mathbb{E}\left[\int_{0}^{T} X_{t}^{\prime} \partial_{x} H^{*}(t) d t\right]-\mathbb{E}\left[\int_{0}^{T} \partial_{\bar{x}} H^{*}(t) X_{t-\delta}^{\prime} d t\right] \\
- & \mathbb{E}\left[\int_{0}^{T} \tilde{\mathbb{E}}\left[\partial_{m} H^{*}(t)\left(\tilde{X}_{t}^{*}\right) \tilde{X}_{t}^{\prime}\right] d t\right]-\mathbb{E}\left[\int_{0}^{T} \tilde{\mathbb{E}}\left[\partial_{\bar{m}} H^{*}(t)\left(\tilde{X}_{t-\delta}^{*}\right) \tilde{X}_{t-\delta}^{\prime}\right] d t\right] \\
& +\mathbb{E}\left[\int_{0}^{T} \int_{0}^{T} q_{s}^{*} \sigma^{\prime}(t) \varphi_{H}(t, s) d t d s\right] .
\end{aligned}
$$

By $(5.1)-5.3)$, we obtain

$$
\begin{aligned}
& J(u)-J\left(u^{*}\right) \\
& \leqslant \mathbb{E}\left[\int_{0}^{T}\left(H(t)-H^{*}(t)\right) d t\right]-\mathbb{E}\left[\int_{0}^{T} \partial_{x} H^{*}(t) X_{t}^{\prime} d t\right]-\mathbb{E}\left[\int_{0}^{T} \partial_{\bar{x}} H^{*}(t) X_{t-\delta}^{\prime} d t\right] \\
& \quad-\mathbb{E}\left[\int_{0}^{T} \tilde{\mathbb{E}}\left[\partial_{m} H^{*}(t)\left(\tilde{X}_{t}^{*}\right) \tilde{X}_{t}^{\prime}\right] d t\right]-\mathbb{E}\left[\int_{0}^{T} \tilde{\mathbb{E}}\left[\partial_{\bar{m}} H^{*}(t)\left(\tilde{X}_{t-\delta}^{*}\right) \tilde{X}_{t-\delta}^{\prime}\right] d t\right] \\
& \leqslant 0 .
\end{aligned}
$$

Due to the concavity assumption on $H$ and because $u^{*}$ satisfies the maximum condition, the first order derivative at $u^{*}$ vanishes.

\section{APPLICATIONS}

The main applications of mean-field dynamics that appear in the literature rely mainly on a dependence upon the probability measures through functions of scalar moments of the measures. More precisely, we assume that 


$$
\begin{aligned}
b(t, x, \bar{x}, m, \bar{m}, u) & =\hat{b}\left(t, x, \bar{x},\left(\psi_{1}, m\right),\left(\psi_{2}, \bar{m}\right), u\right) \\
\sigma(t, m, \bar{m}) & =\hat{\sigma}\left(t,\left(\phi_{1}, m\right),\left(\phi_{2}, \bar{m}\right)\right) \\
f(t, x, \bar{x}, m, \bar{m}, u) & =\hat{f}\left(t, x, \bar{x},\left(\gamma_{1}, m\right),\left(\gamma_{2}, \bar{m}\right), u\right) \\
g(x, m) & =\hat{g}\left(x,\left(\gamma_{3}, m\right)\right)
\end{aligned}
$$

for some scalar functions $\psi_{1}, \psi_{2}, \phi_{1}, \phi_{2}, \gamma_{1}, \gamma_{2}, \gamma_{3}$ with at most quadratic growth at $\infty$. The functions $\hat{b}, \hat{f}$ are defined on $[0, T] \times \mathbb{R} \times \mathbb{R} \times \mathbb{R} \times \mathbb{R} \times \mathcal{U}$, the function $\hat{\sigma}$ is defined on $[0, T] \times \mathbb{R} \times \mathbb{R}$ and $\hat{g}$ is defined on $\mathbb{R} \times \mathbb{R}$. The notation $(\psi, m)$ denotes the integral of the function $\psi$ with respect to the probability measure $m$. The Hamiltonian that we defined in the previous section takes now the following form:

$$
\begin{aligned}
& H(t, x, \bar{x}, m, \bar{m}, u, p, q(\cdot))=\hat{f}\left(t, x, \bar{x},\left(\gamma_{1}, m\right),\left(\gamma_{2}, \bar{m}\right), u\right) \\
& \quad+p \hat{b}\left(t, x, \bar{x},\left(\psi_{1}, m\right),\left(\psi_{2}, \bar{m}\right), u\right)+\hat{\sigma}\left(t,\left(\phi_{1}, m\right),\left(\phi_{2}, \bar{m}\right)\right) \int_{0}^{T} q(s) \varphi_{H}(s, t) d s .
\end{aligned}
$$

According to the definition of differentiability with respect to measures recalled in the preliminaries, the derivative of the Hamiltonian with respect to the measure $m$, for instance, is computed as follows:

$$
\begin{aligned}
\partial_{m} H(t)\left(x^{\prime}\right)= & \partial_{x^{\prime}} \hat{f}\left(t, x, \bar{x},\left(\gamma_{1}, m\right),\left(\gamma_{2}, \bar{m}\right), u\right) \gamma_{1}^{\prime}\left(x^{\prime}\right) \\
& +p \times \partial_{x^{\prime}} \hat{b}\left(t, x, \bar{x},\left(\psi_{1}, m\right),\left(\psi_{2}, \bar{m}\right), u\right) \psi_{1}^{\prime}\left(x^{\prime}\right) \\
& +\partial_{x^{\prime}} \hat{\sigma}\left(t,\left(\phi_{1}, m\right),\left(\phi_{2}, \bar{m}\right)\right) \phi_{1}^{\prime}\left(x^{\prime}\right) \int_{0}^{T} q(s) \varphi_{H}(s, t) d s
\end{aligned}
$$

where $\partial_{m} H(t)\left(x^{\prime}\right)=\partial_{m} H(t, x, \bar{x}, m, \bar{m}, u, p, q(\cdot))\left(x^{\prime}\right)$. The terminal value of the adjoint BSDE (4.6), which is $p_{T}=\partial_{x} g(T)+\tilde{\mathbb{E}}\left[\partial_{m} \tilde{g}(T)\left(X_{T}\right)\right]$, can be written in terms of the derivatives of the function $\hat{g}$ as follows:

$$
p_{T}=\partial_{x} \hat{g}\left(X_{T}, \mathbb{E}\left[\gamma_{3}\left(X_{T}\right)\right]\right)+\tilde{\mathbb{E}}\left[\partial_{x^{\prime}} \hat{g}\left(\tilde{X}_{T}, \mathbb{E}\left[\gamma_{3}\left(X_{T}\right)\right)\right] \gamma_{3}^{\prime}\left(X_{T}\right),\right.
$$

where $\tilde{X}_{T}$ is an independent copy of $X_{T}$. The following two applications illustrate the previous results.

6.1. Optimal consumption from a cash flow with delay. We consider the problem of optimal consumption with a cash flow $X:=X^{\rho}$ whose dynamic is related to its value at previous time instants, namely, we assume that $X$ satisfies the following SDE:

$$
\left\{\begin{array}{l}
d X_{t}=\left[\mathbb{E}\left[X_{t-\delta}\right]-\rho(t)\right] d t+\beta(t) d B_{t}^{H}, \quad t \in[0, T] \\
X_{t}=x_{0}(t), \quad t \in[-\delta, 0] .
\end{array}\right.
$$


where $\rho$ is the relative consumption rate (our control), $x_{0}$ a bounded deterministic function, $\delta$ a strictly positive constant and $\beta$ a given deterministic function in $\mathbb{L}_{H}^{2}([0, T])$. The integral with respect to the $\mathrm{fBm}$ is therefore a Wiener type integral.

This model typically represents the evolution of the operating activities of an organization when incomings and outgoings of cash occur with a delay.

The problem we consider is to find the consumption rate $\rho^{*} \in \overline{\mathcal{A}}_{\mathcal{F}}$ such that

$$
J\left(\rho^{*}\right)=\sup _{\rho \in \overline{\mathcal{A}}_{\mathcal{F}}} J(\rho)
$$

where

$$
J(\rho)=\mathbb{E}\left[\int_{0}^{T} \log (\rho(t)) d t+\xi_{1} \mathbb{E}\left[X_{T}\right]^{2}\right],
$$

over the set $\overline{\mathcal{A}}_{\mathcal{F}}$ of admissible controls which are $\mathcal{F}$-adapted processes with values in $\mathbb{R}_{+}^{*}, \xi_{1}>0$ is a given bounded $\mathcal{F}_{T}$-measurable random variable assumed to be in $\mathcal{L}_{H}^{1,2}([0, T])$, and $\xi_{1}$ represents a random effect influencing the terminal cost functional. We also assume that $X$ exists and belongs to $L^{2}(\Omega \times[0, T])$.

Note that the running cost functional we consider in this example is the function $\rho(t) \mapsto \log (\rho(t))$ which represents the utility function of the organization. Moreover, in order to control the random fluctuations of the terminal value of the cash flow $X_{T}^{\rho}$, we chose to introduce the square of its mean in the terminal cost functional. Therefore according to the notations used in the previous section, the terminal cost functional is of mean-field type, more precisely it has the form

$$
g\left(X_{T}, \mathbb{P}_{X_{T}}\right)=g\left(\mathbb{P}_{X_{T}}\right)=\xi_{1} \mathbb{E}\left[X_{T}\right]^{2}=\hat{g}_{\xi_{1}}\left(\left(\mathrm{ID}, \mathbb{P}_{X_{T}}\right)\right)
$$

where $\hat{g}_{\xi_{1}}(x)=\xi_{1} x^{2}$, therefore $\hat{g}_{\xi_{1}}^{\prime}(x)=2 \xi_{1} x$ a.s.

The Hamiltonian of this control problem is given by

$$
H(t, x, \bar{m}, \rho, p, q(\cdot))=\log (\rho)+((\mathrm{ID}, \bar{m})-\rho) p+\beta(t) \int_{0}^{T} q(s) \varphi_{H}(s, t) d s,
$$

where $(p, q)$ is the solution of the associated adjoint BSDE

$$
\left\{\begin{array}{l}
d p_{t}=-\tilde{\mathbb{E}}\left[\tilde{p}_{t+\delta} \chi_{[0, T-\delta]}(t)\right] d t+q_{t} d B_{t}^{H}, \quad t \in[0, T], \\
p_{T}=2 \xi_{1} \mathbb{E}\left[X_{T}\right] .
\end{array}\right.
$$

Inspired by the resolution of the linear BSDE driven by a fractional Brownian motion with Hurst parameter $H>1 / 2$ in [2] and [13], we propose a resolution of the anticipated BSDE (6.2) by solving a sequence of linear BSDEs following this procedure: 
STEP 1. If $t \in[T-\delta, T]$, the previous BSDE takes the form

$$
\left\{\begin{array}{l}
d p_{t}=q_{t} d B_{t}^{H}, \quad t \in[T-\delta, T], \\
p_{T}=2 \xi_{1} \mathbb{E}\left[X_{T}\right] .
\end{array}\right.
$$

which has a unique solution according to the resolution in [13] and [2], given explicitly by

$$
p_{t}=2 \mathbb{E}\left[X_{T}\right] \hat{\mathbb{E}}\left[\xi_{1} \mid \mathcal{F}_{t}\right], \quad q_{t}=2 \mathbb{E}\left[X_{T}\right] \hat{\mathbb{E}}\left[D_{t}^{H} \xi_{1} \mid \mathcal{F}_{t}\right], \quad t \in[T-\delta, T] .
$$

Existence and uniqueness of $q$ follow from [11, Theorem 12.1], and $\hat{\mathbb{E}}$ is the quasiconditional expectation (see [3] for the definition).

STEP 2. If $t \in[T-2 \delta, T-\delta]$ and $T-2 \delta>0$, using the fact that $\tilde{p}$ and $p$ have the same law, we obtain the BSDE

$$
\left\{\begin{array}{l}
d p_{t}=-\mathbb{E}\left[p_{t+\delta}\right] d t+q_{t} d B_{t}^{H}, \quad t \in[T-2 \delta, T-\delta], \\
p_{T-\delta} \quad \text { known from Step } 1 .
\end{array}\right.
$$

As in Step 1, this BSDE has an explicit unique solution given by

$$
p_{t}=\hat{\mathbb{E}}\left[p_{T-\delta}+\int_{t}^{T} \theta(s) d s \mid \mathcal{F}_{t}\right], \quad q_{t}=D_{t}^{H} p_{t}, \quad t \in[T-2 \delta, T-\delta]
$$

where $\theta(t)=\mathbb{E}\left[p_{t+\delta}\right]$ and $p_{t+\delta}$ is known by Step 1 .

We continue like this by induction up to and including Step $n$, where $n$ is such that $T-n \delta \leqslant 0<T-(n-1) \delta$ and we solve the corresponding BSDE on the time interval $[0, T-(n-1) \delta]$.

Maximizing $H$ with respect to $\rho$ gives the following first order condition for an optimal consumption rate $\rho^{*}$ :

$$
\partial_{\rho} H^{*}(t)=\frac{1}{\rho^{*}(t)}-p_{t}^{*}=0
$$

If

$$
p^{*}(t)>0 \quad \text { for all } t \in[0, T]
$$

we get

$$
\rho^{*}(t)=\frac{1}{p_{t}^{*}} \quad \text { for all } t \in[0, T],
$$

where $p$ satisfies the previous anticipated BSDE.

THEOREM 6.1. Let $(p, q)$ be the solution of the BSDE (6.2) and suppose that (6.3) holds. Then any optimal consumption rate $\rho^{*}$ satisfies (6.4) and the corresponding optimal cash flow $X^{*}$ is given by (6.1). 
6.2. Linear-quadratic problem with delay. We now consider a linear-quadratic (LQ) model for a controlled process $X=X^{\alpha}$ given by the following delayed stochastic differential equation:

$$
\left\{\begin{array}{l}
d X_{t}=\left[\beta_{1}(t) X_{t-\delta}+\alpha(t)\right] d t+\beta_{2}(t) d B_{t}^{H}, \quad t \in[0, T] \\
X_{t}=x_{0}(t), \quad t \in[-\delta, 0]
\end{array}\right.
$$

where $\delta>0$ is a given constant, $\beta_{1}, x_{0}$ are given bounded deterministic functions, and $\beta_{2}$ is a given deterministic function in $\mathbb{L}_{H}^{2}([0, T])$. The integral with respect to the fBm is therefore a Wiener type integral and $\alpha \in \mathcal{A}_{\mathcal{F}}$ is our control process, where the set $\mathcal{A}_{\mathcal{F}}$ consists of the admissible controls assumed to be square integrable $\mathcal{F}$-adapted processes with real values.

We want to minimize the expected value of $\left(X_{T}-\mathbb{E}\left[X_{T}\right]\right)^{2}$ which is the variance of $X_{T}$ with a minimal average use of energy, measured by the integral $\mathbb{E}\left[\int_{0}^{T} \alpha_{t}^{2} d t\right]$, more precisely, the performance functional is of the following form:

$$
J(\alpha)=-\frac{1}{2}\left(\operatorname{VAR}\left(X_{T}\right)+\mathbb{E}\left[\int_{0}^{T} \alpha_{t}^{2} d t\right]\right) .
$$

Our goal is therefore to find a control process $\alpha^{*} \in \mathcal{A}_{\mathcal{F}}$ such that

$$
J\left(\alpha^{*}\right)=\sup _{\alpha \in \mathcal{A}_{\mathcal{F}}} J(\alpha) .
$$

Including the variance of the state process in the cost functional in order to keep it small is a way to control its sensitivity to possible variations of random events. The form of this cost functional is inspired by [20].

Note that the terminal cost functional of our problem has the form

$$
g\left(X_{T}, \mathbb{P}_{X_{T}}\right)=\hat{g}\left(X_{T},\left(\mathrm{ID}, \mathbb{P}_{X_{T}}\right)\right)=-\frac{1}{2}\left(X_{T}-\mathbb{E}\left[X_{T}\right]\right)^{2},
$$

where $\hat{g}\left(x, x^{\prime}\right)=-\frac{1}{2}\left(x-x^{\prime}\right)^{2}$, therefore $\partial_{x} \hat{g}\left(x, x^{\prime}\right)=-\left(x-x^{\prime}\right)=-\partial_{x^{\prime}} \hat{g}\left(x, x^{\prime}\right)$. Therefore the terminal value of the solution of the adjoint BSDE is

$$
\begin{aligned}
p_{T} & =\partial_{x} \hat{g}\left(X_{T},\left(\mathrm{ID}, \mathbb{P}_{X_{T}}\right)\right)+\tilde{\mathbb{E}}\left[\partial_{x^{\prime}} \hat{g}\left(\tilde{X}_{T},\left(\mathrm{ID}, \mathbb{P}_{X_{T}}\right)\right)\right] \\
& =-\left(X_{T}-\mathbb{E}\left[X_{T}\right]\right)+\tilde{\mathbb{E}}\left[\tilde{X}_{T}-\mathbb{E}\left[X_{T}\right]\right]=-\left(X_{T}-\mathbb{E}\left[X_{T}\right]\right),
\end{aligned}
$$

where we use the fact that $\tilde{X}$ and $X$ have the same distribution.

The Hamiltonian of our control problem takes the following form:

$$
H(t, x, \bar{x}, \alpha, p, q(\cdot))=-\frac{1}{2} \alpha^{2}+\left(\beta_{1}(t) \bar{x}+\alpha\right) p+\beta_{2}(t) \int_{0}^{T} q_{s} \varphi_{H}(s, t) d s
$$


where $(p, q)$ is the solution of the associated adjoint BSDE:

$$
\left\{\begin{array}{l}
d p_{t}=-\beta_{1}(t+\delta) \mathbb{E}\left[p_{t+\delta} \chi_{[0, T-\delta]}(t) \mid \mathcal{F}_{t}\right] d t+q_{t} d B_{t}^{H}, \quad t \in[0, T], \\
p_{T}=-\left(X_{T}-\mathbb{E}\left[X_{T}\right]\right) .
\end{array}\right.
$$

This BSDE can be solved as in the previous example by solving a sequence of linear BSDEs. The resolution by steps gives an explicit unique solution; we omit the details since they are similar to the first example. On the other hand, the function $\alpha \mapsto H\left(t, X_{t}, X_{t-\delta}, \alpha_{t}, p_{t}, q(\cdot)\right)$ is maximal when

$$
\alpha_{t}=\alpha_{t}^{*}=p_{t}^{*}
$$

where $p^{*}$ satisfies

$$
\left\{\begin{array}{l}
d p_{t}^{*}=-\beta_{1}(t+\delta) \mathbb{E}\left[p_{t+\delta}^{*} \chi_{[0, T-\delta]}(t) \mid \mathcal{F}_{t}\right] d t+q_{t}^{*} d B^{H}(t), \quad t \in[0, T] \\
p_{T}=-\left(X_{T}^{*}-\mathbb{E}\left[X_{T}^{*}\right]\right)
\end{array}\right.
$$

Thus, we have proved the following theorem.

THEOREM 6.2. The optimal control $\alpha^{*}$ of the LQ problem (6.7) is given by (6.8), where $\left(X^{*}, p^{*}, q^{*}\right)$ solves the couple of systems (6.5) and (6.9) of forwardbackward stochastic differential equations.

\section{REFERENCES}

[1] D. Andersson and B. Djehiche, A maximum principle for SDEs of mean-field type, Appl. Math. Optim. 63 (2011), 341-356.

[2] F. Biagini, Y. Hu and B. Øksendal, A stochastic maximum principle for processes driven by fractional Brownian motion, Stochastic Process. Appl. 100 (2002), 233-253.

[3] F. Biagini, Y. Hu, B. Øksendal and T. S. Zhang, Stochastic Calculus for Fractional Brownian Motion and Applications, Springer, London, 2008.

[4] R. Buckdahn and S. Jing, Mean-field SDE driven by a fractional Brownian motion and related stochastic control problem, SIAM J. Control Optim. 55 (2017), 1500-1533.

[5] R. Buckdahn, J. Li and S. Peng, Mean-field stochastic differential equations and associated PDEs, Ann. Probab. 45 (2017), 824-878.

[6] P. Cardaliaguet, Notes on mean field games, preprint, Univ. Paris Dauphine, 2013.

[7] R. Carmona and F. Delarue, Control of McKean-Vlasov dynamics versus mean field games, Math. Finance Econom. 7 (2013), 131-166.

[8] R. Carmona and F. Delarue, Forward-backward stochastic differential equations and controlled McKean-Vlasov dynamics, Ann. Probab. 43 (2015), 2647-2700.

[9] R. Carmona, J.-P. Fouque, S. M. Mousavi and L. H. Sun, Systemic risk and stochastic games with delay, J. Optim. Theory Appl. 179 (2018), 366-399.

[10] $\mathrm{L}$. Chen and $\mathrm{Z}$. Wu, Maximum principle for the stochastic optimal control problem with delay and application, Automatica 46 (2010), 1074-1080.

[11] Y. Hu, Integral transformations and anticipative calculus for fractional Brownian motions, Mem. Amer. Math. Soc. 175 (2005), no. 825, 127 pp.

[12] Y. Hu and B. Øksendal, Fractional white noise calculus and applications to finance, Infin. Dimens. Anal. Quantum Probab. Related Topics 6 (2003), 1-32. 
[13] Y. Hu and S. Peng, Backward stochastic differential equation driven by fractional Brownian motion, SIAM J. Control Optim. 48 (2009), 1675-1700.

[14] Y. Hu and X. Y. Zhou, Stochastic control for linear systems driven by fractional noises, SIAM J. Control Optim. 43 (2005), 2245-2277.

[15] J.-M. Lasry and P.-L. Lions, Mean field games, Japan. J. Math. 2 (2007), 229-260.

[16] P.-L. Lions, Cours au Collège de France, www.college-de-france.fr.

[17] B. Øksendal and A. Sulem, A maximum principle for optimal control of stochastic systems with delay, with applications to finance, in: Optimal Control and Partial Differential EquationsInnovations and Applications, J. M. Menaldi et al. (eds.), IOS Press, Amsterdam, 2001, 64-79.

[18] B. Øksendal, A. Sulem and Z. Thusheng, Optimal control of stochastic delay equations and time-advanced backward stochastic differential equations, Adv. Appl. Probab. 43 (2011), 572-596.

[19] Q. Wang, F. Chen and F. Huang, Maximum principle for optimal control problem of stochastic delay differential equations driven by fractional Brownian motions, Optim. Control Appl. Methods 37 (2016), 90-107.

[20] J. Yong, Linear-quadratic optimal control problems for mean-field stochastic differential equations, SIAM J. Control Optim. 51 (2013), 2809-2838.

Soukaina Douissi

Laboratory LIBMA, Faculty Semlalia

University Cadi Ayyad

Boulevard Prince My Abdellah

40000 Marrakech, Morocco

E-mail: douissi.soukaina@gmail.com

Astrid Hilbert

Department of Mathematics

Linnaeus University

SE-351 95 Växjö, Sweden

E-mail: astrid.hilbert@lnu.se
Nacira Agram

Department of Mathematics

University of Oslo

P.O. Box 1053 Blindern

$\mathrm{N}-0316$ Oslo, Norway

E-mail: naciraa@math.uio.no

Received 6.3.2018;

revised version 9.1.2019 